\title{
Yolk sac-derived Pdcd11-positive cells modulate zebrafish microglia differentiation through the NF-KB-Tgf $\beta 1$ pathway
}

\author{
Ruimeng Yang ${ }^{1,2}$ - Ming Zhan ${ }^{2,3} \cdot$ Miaomiao Guo ${ }^{2}$ Hao Yuan ${ }^{1} \cdot$ Yiqin Wang ${ }^{1}$ - Yiyue Zhang $\mathbb{D}^{4} \cdot$ Wenqing Zhang ${ }^{4}$. \\ Saijuan Chen ${ }^{1} \cdot$ Hugues de The $\mathbb{1}^{1,5,6} \cdot$ Zhu Chen $^{1} \cdot$ Jun Zhou ${ }^{1} \cdot$ Jun Zhu ${ }^{1,5}$
}

Received: 3 March 2020 / Revised: 7 July 2020 / Accepted: 9 July 2020 / Published online: 24 July 2020

(c) The Author(s) 2020. This article is published with open access

\begin{abstract}
Microglia are the primary immune cells in the central nervous system, which plays a vital role in neuron development and neurodegenerative diseases. Microglial precursors in peripheral hematopoietic tissues colonize the central nervous system during early embryogenesis. However, how intrinsic and extrinsic signals integrate to regulate microglia's differentiation remains undefined. In this study, we identified the cerebral white matter hyperintensities susceptibility gene, programmed cell death protein 11 (PDCD11), as an essential factor regulating microglia differentiation. In zebrafish, pdcd11 deficiency prevents the differentiation of the precursors to mature brain microglia. Although, the inflammatory featured macrophage brain colonization is augmented. At $22 \mathrm{~h}$ post fertilization, the Pdcd11-positive cells on the yolk sac are distinct from macrophages and neutrophils. Mechanistically, PDCD11 exerts its physiological role by differentially regulating the functions of nuclear factor-kappa B family members, P65 and c-Rel, suppressing P65-mediated expression of inflammatory cytokines, such as tnf $\alpha$, and enhancing the c-Rel-dependent appearance of tgf $\beta 1$. The present study provides novel insights in understanding microglia differentiation during zebrafish development.
\end{abstract}

\section{Introduction}

Cerebral white matter hyperintensities (WMH), also known as leukoaraiosis, are common in the aging population and are

These authors contributed equally: Ruimeng Yang, Ming Zhan

Edited by M. Bianchi

Supplementary information The online version of this article (https:// doi.org/10.1038/s41418-020-0591-3) contains supplementary material, which is available to authorized users.

Jun Zhou

junjun_j@yahoo.com

$\triangle$ Jun Zhu

zhuj1966@yahoo.com

1 CNRS-LIA Hematology and Cancer, Sino-French Research Center for Life Sciences and Genomics, State Key Laboratory of Medical Genomics, Ruijin Hospital, Shanghai Jiao Tong University School of Medicine, 200025 Shanghai, PR China

2 The Core Laboratory in Medical Center of Clinical Research, Department of Endocrinology, Shanghai Ninth People's Hospital, State Key Laboratory of Medical Genomics, Shanghai Jiao Tong University School of Medicine, 200011 Shanghai, PR China associated with increased risk of cognitive dysfunction, stroke, and neurodegenerative disorders including Alzheimer's disease [1]. Perivascular inflammation is a prominent pathological feature in $\mathrm{WMH}$, and $\mathrm{WMH}$ burden has been associated with circulating biomarkers of inflammation, including highsensitivity C-reactive protein and Interleukin-6 [2-4]. Genome-Wide Association studies have helped identify many risk-associated single nucleotide polymorphisms (SNPs) related with WMH development and progression [5-7]. SNPs in PDCD11 have showed significant associations with $\mathrm{WMH}$ burden, but their functional role remain undefined $[5,7]$.

3 Department of Urology, Shanghai Ninth People's Hospital, Shanghai Jiao Tong University School of Medicine, 200011 Shanghai, PR China

4 Division of Cell, Developmental and Integrative Biology, School of Medicine, South China University of Technology, 510006 Guangzhou, PR China

5 Université de Paris 7/INSERM/CNRS UMR 944/7212, Equipe Labellisée No. 11 Ligue Nationale Contre le Cancer, Hôpital St. Louis, Paris, France

6 Chaire d'Oncologie Cellulaire et Molecular, College de France, PSL Universite, Paris, France 
A

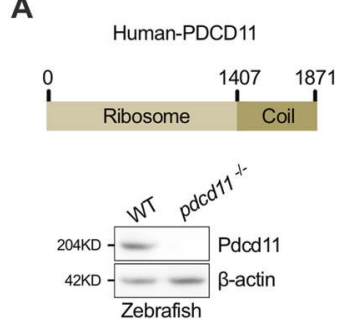

E
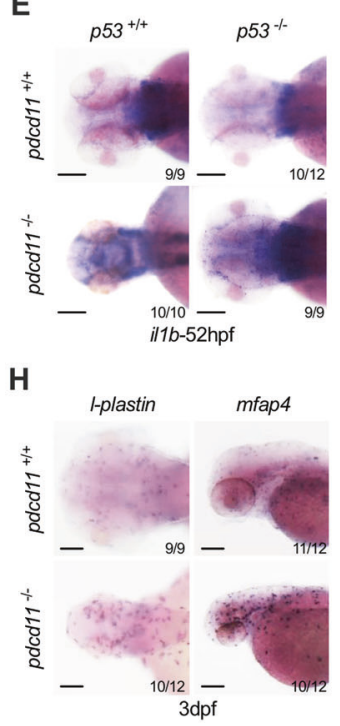

B

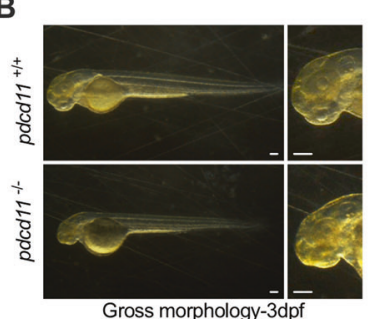

$\mathbf{F}$
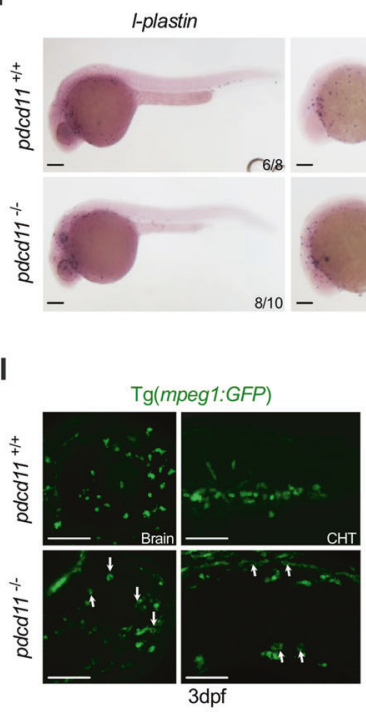

C

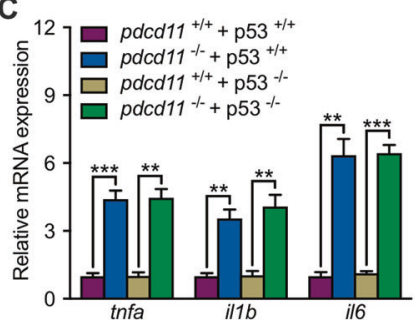

mfap4

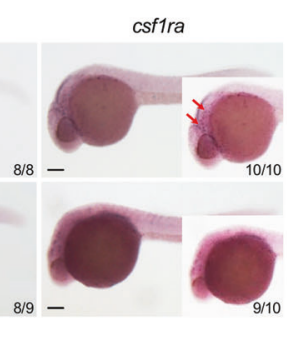

$\mathrm{J}$

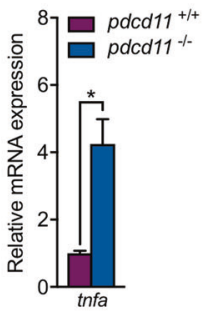

$\mathrm{K}$

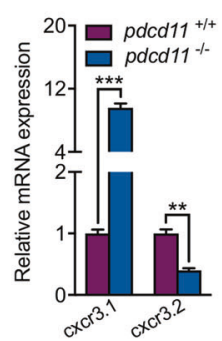

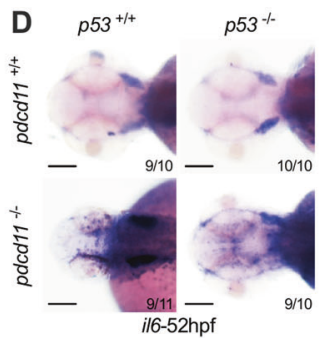

G

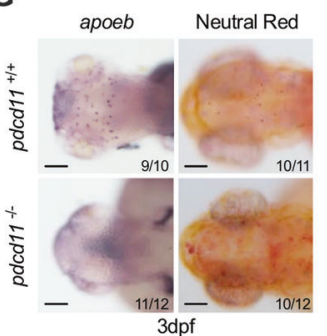

$\mathbf{L}$

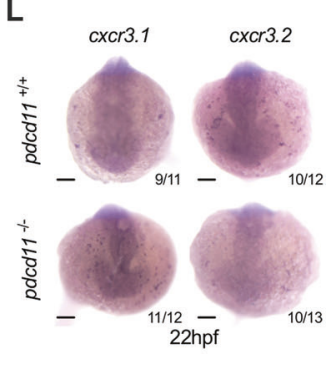

Fig. 1 Pdcd11 mutant zebrafish favor inflammatory macrophage generation. a Schematic diagram showing the two major function domains of PDCD11: N-terminal Ribosome domain (1-1407) and CTerminal Coil domain (1408-1871). b Gross morphology of 3 dpf WT and pdcd11 mutants. c qPCR confirmation of hyperactivated inflammatory pathway genes, including tnfa, illb, and il6, in $22 \mathrm{hpf} p d c d 11$ mutants, which could not be restored by combined $p 53$ mutation. d-e $I l 6$ and $i l 1 b$ expression in the brain of WT or pdcd11 mutants with $p 53$ mutated or not examined by WISH at $52 \mathrm{hpf}$. f WISH analysis of $l$ plastin, mfap4, and csflra expression in $24 \mathrm{hpf}$ WT and pdcd11 mutant embryos. Red arrows indicate csfl ra-positive cells in the brain. g Microglia development in $3 \mathrm{dpf}$ WT and pdcd11 mutants by WISH assessment of apoeb and Neutral Red staining. h L-plastin and mfap4 expression pattern in $3 \mathrm{dpf}$ WT and pdcd11 mutants. i Morphology of

Microglia, the innate immune cells of the central nervous system (CNS), enter the brain during early embryogenesis and play important roles in white matter pathology [8]. As influenced by their environment, microglia assume different molecular patterns ranging from M1 phenotype in secretion pro-inflammatory cytokines to M2 phenotype in inflammation resolution and tissue repair [9]. In the zebrafish embryo, macrophages develop early on the yolk sac (YS) expression L-plastin and spread in the cephalic mesenchyme at about $24 \mathrm{~h}$ post fertilization (hpf). After which they invaded brain and differentiated into microglia featured by lower expression of the L-plastin, and higher expression of the neurotrophic lipid carrier, apolipoprotein E at about $60 \mathrm{hpf}$ $[10,11]$. Factors like Colony-Stimulating Factor 1 Receptor macrophages in the brain and caudal hematopoietic tissue (CHT) were examined using the $\operatorname{Tg}($ mpeg $1: G F P)$ transgenic line. White arrows indicate the vacuolated macrophages found in the pdcd11 mutant. j Increased tnfa mRNA expression in sorted macrophages $\mathrm{Tg}$ (mpeg1: GFP) from $60 \mathrm{hpf} p d c d 11$ mutants as compared with WT controls. $\mathbf{k}$ qPCR examination of the expression of macrophage-related genes cxcr3.1 and cxcr3.2 in 22 hpf WT and pdcd11 mutants. I WISH analysis of $c x c r 3.1$ and $c x c r 3.2$ expression in $22 \mathrm{hpf}$ WT and pdcd11 mutants. The number positioned in the lower right corner of $\mathbf{d}-\mathbf{h}, \mathbf{I}$ represent the number of zebrafish embryos shown positive phenotypes versus the total number of embryos examined. Scale bar: $100 \mu \mathrm{m}$. Black horizontal lines indicate mean \pm standard error of mean (SEM). Means \pm SEM are shown for three independent experiments. ${ }^{*} P<0.05 ;{ }^{* *} P<0.01 ;{ }^{* * *} P<0.001$ (Student's $t$ test).

(CSF1R) and neuronal apoptosis modulate the seeding of microglia precursors in the brain $[12,13]$. But how intrinsic and extrinsic signals integrate in microglia differentiation remains largely unknown. Transforming growth factor-beta (TGF- $\beta$ ) family members are cytokine important for both innate and adaptive immune functions [14]. It is required for the maintenance of the microglia-specific homeostatic gene signature. Deletion of the TGF- $\beta$ receptor in microglia resulted in rapid conversion of microglia toward an inflammatory macrophage phenotype $[15,16]$. Although the importance of brain-derived TGF- $\beta 1$ in shaping immune response in pathogenic conditions has been demonstrated, the origin of embryonic TGF- $\beta 1$, especially for inducing microglia differentiation, remains unknown. 
In this study, helped by zebrafish model organism, we demonstrated that PDCDI1, a WHM susceptibility gene, helps promote embryonic microglia differentiation through Tgf $\beta 1$. Macrophages in $p d c d 11$ mutants showed increased expression of inflammatory genes and impaired upregulation of microglia signature genes. This imbalanced macrophage generation, likely caused by uncontrolled P65 activation, causes inflammatory gene upregulation and suppresses c-Rel-dependent Tgf $\beta 1$ expression under $p d c d 11$ deficiency.

\section{Results}

\section{P53 independent inflammatory pathway activation in pdcd11 mutants}

Pdcd11 is highly conserved between species and mainly harbors two functional domains, including an N-terminal ribosome and Coil (Fig. 1a). To define the role of Pdcd11 in embryonic development, we generated a $p d c d 11$ mutant zebrafish line with a 7-bp deletion in the fourth exon by CRISPR-Cas9 nuclease (Fig. S1a). Antibody targeting the ribosome domain of Pdcd11 confirmed the complete loss of functional domains in $p d c d 11$ mutants (Fig. 1a). Perturbations of ribosome biogenesis often stabilize and activate genes related to the P53 apoptosis pathway [17-19]. Common with other reported ribosome defects, obvious developmental retardation and craniofacial malformation were observed in $3 \mathrm{dpf} p d c d 11$ mutants (Fig. 1b). Not surprisingly, P53 pathway genes were hyperactivated (Fig. S1b) and acridine orange (AO)-positive dead cells were also significantly increased in $p d c d l 1$ deficient embryos from $22 \mathrm{hpf}$ (Fig. S1c). To determine the contribution of the induction of apoptosis in the developmental defects of $p d c d 11$ mutants, $p d c d 11$ mutants in a $p 53$ knockout background were also generated [20]. P53 depletion favorably recovered AO cell numbers and P53 pathway genes in pdcdl1 mutants (Fig. S1d, e). However, cranial deformities, shorter body lengths, and survival rates were only partially restored (Fig. S1f, g), possibly suggesting that other P53 independent factors also contribute to the pathology of $p d c d 11$ mutants.

Interestingly, consistent with the perivascular inflammation a prominent pathological feature in $\mathrm{WMH}$, we found that genes associated with inflammatory responses, like il6, $i l l b$, and tnfa, were significantly increased, which otherwise failed to be restored by combined $p 53$ mutation (Fig. 1c). Using whole-mount in situ hybridization (WISH), the apparent increased expression of inflammatory genes in the brain of $52 \mathrm{hpf} p d c d 11$ mutants were detected, which also could not be recovered by the $p 53$ mutation (Fig. 1d, e).

\section{Macrophage generation imbalance in pdcd11 mutants}

Inflammatory gene expression was elevated in $p d c d 11$ mutants as early as $22 \mathrm{hpf}$. At this time, macrophages and neutrophils were the two major types of immune-related inflammatory cells identified in zebrafish. Macrophages initiate migration in the cephalic mesenchyme at about 24 hpf. Compared with that in WT embryos, $p d c d 11$ deficiency induced increased and earlier accumulation of macrophages in brain tissue as revealed by mfap 4 or pan-leukocyte marker l-plastin staining (Fig. 1f). The distribution pattern of mpo-positive leukocytes remained unaltered at this time (Fig. S2a). A previous study showed that apoptotic neuronal death drives the colonization of microglia precursors in the optic tectum [21]. Thus, we speculated that the advanced macrophage migration might be stimulated by increased neural apoptotic signaling. However, the combined $p 53$ mutation failed to restore the increased macrophage accumulation in the brains of $p d c d l 1$ mutants, with accumulation actually being exacerbated (Fig. S2b). Interestingly, distinct from nonspecific macrophage markers, the expression of csflra, which was previously shown to be required for the migration and proliferation of microglia precursors $[13,22]$, was contrarily reduced in pdcdll mutants (Fig. 1f). Consistently, staining by either apoeb or Neutral Red revealed that mature microglia, which are tissueresident macrophages in the brain, were completely absent in $3 \mathrm{dpf} p d c d 11$ mutants (Fig. 1g). At this time, macrophages stained for mfap4 or l-plastin were abundant in the brain of $p d c d 11$ mutants, with an evidently enlarged and vacuolated morphology, which was also verified using a macrophage transgenic line $\operatorname{Tg}($ mpeg 1:GFP) (Fig. 1h, i). Macrophages acquire specific functional phenotypes that are manifest by secretion of inflammatory gene products and their morphological appearance. We found that the expression of tnfa, a well-established marker of M1 macrophages in zebrafish [23], was obviously increased in macrophages sorted from $60 \mathrm{hpf} p d c d 11$-deficient embryos (Fig. 1j). In teleost fish, cxcr3.1 and cxcr3.2 macrophages show functional polarization towards M1 and M2, respectively [24]. We found that in $22 \mathrm{hpf} p d c d 11$ mutants, cxcr3.1 expression was profoundly increased while that of cxcr3.2 was reduced as compared with that in WT controls (Fig. 1k, 1).

Similar to the failed restoration of inflammatory genes with $p 53$ mutation, simply inhibiting apoptosis could not restore the macrophage generation abnormalities observed in pdcd11 mutant embryos (Fig. S2c, d). Moreover, no obvious aberrations in proliferation or apoptosis of myeloid progenitors were detected in pdcdl1 mutants at $22 \mathrm{hpf}$ (Fig. S2e-h). 
A

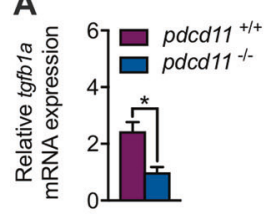

E
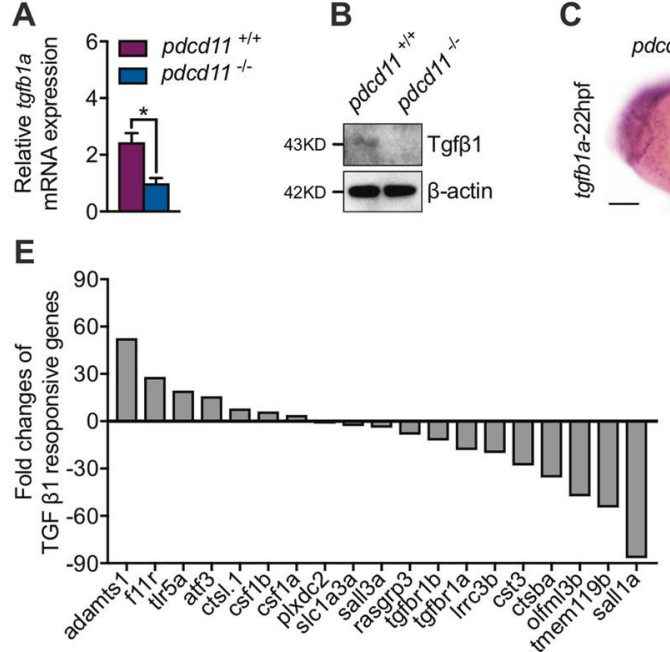

\section{I}

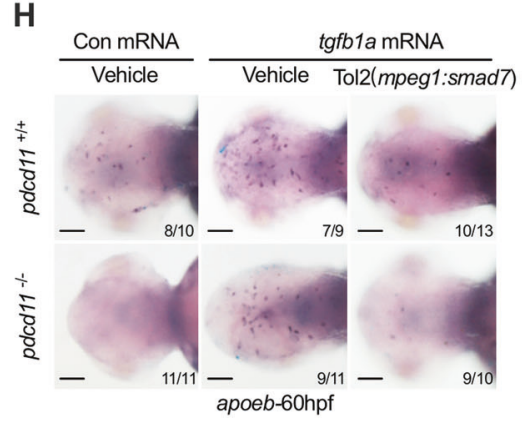

C

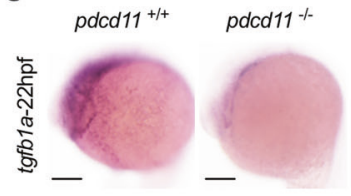

$\mathbf{F}$

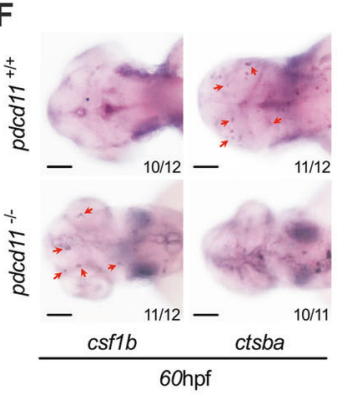

D

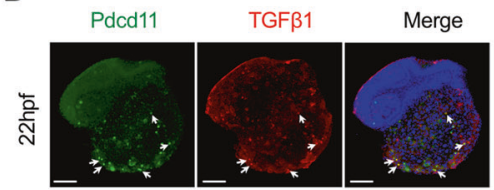

G

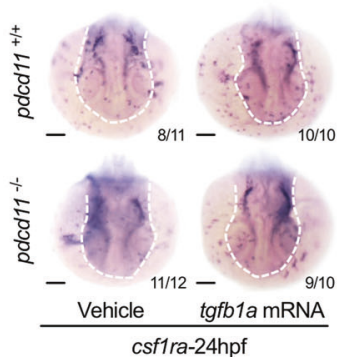

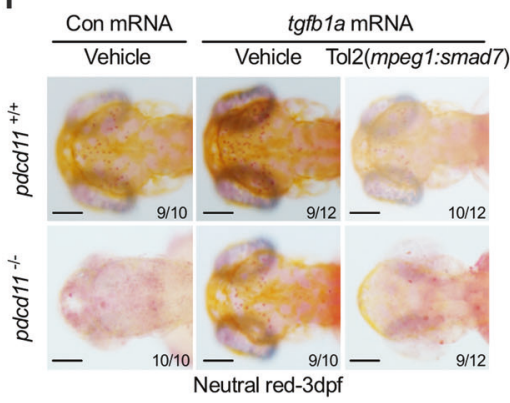

$\mathrm{J}$ $\frac{\text { Con mRNA }}{\text { Vehicle }} \frac{\text { tgfb1a mRNA }}{\text { Vehicle } \quad \text { LY364947 }}$

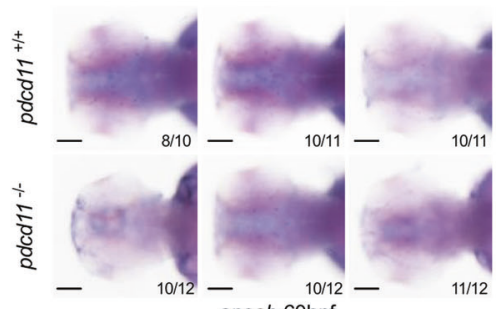

Fig. 2 Reduced TGF- $\beta 1$ signature genes in pdcd11-deficient macrophages. qPCR (a), western blot (b) and WISH (c) examination of the expression levels of tgfbla or Tgf $\beta 1$ in $22 \mathrm{hpf}$ zebrafish. d Immunofluorescence assessment of Pdcd11 and Tgf $\beta 1$ expression in $22 \mathrm{hpf}$ zebrafish embryos. White arrows indicate the co-expressed signals found on the YS. e Fold changes of Tgf $\beta 1$-regulated genes expression in macrophages sorted from the brains of $60 \mathrm{hpf} p d c d 11$ mutants as compared with WT controls using $\operatorname{Tg}($ mpeg $1: G F P)$ transgenic line. f. Expression of $c s f 1 b$ and $c t s b a$ in $60 \mathrm{hpf}$ WT and pdcdl1 mutants by WISH. Red arrows indicate the positive cells. $\mathbf{g}$ Effect of zebrafish tgfbla mRNA overexpression on csflra expression in WT and $p d c d 11$ mutants. Effect of tgfbla mRNA or combined mpegl driven smad7 overexpression on microglia markers apoeb (h) or Neutral Red staining (i) in WT and pdcd11 mutants. $\mathbf{j}$ The effect of LY364947 addition on apoeb expression in WT and pdcd11 mutants with tgfbla mRNA overexpression. The number positioned in the lower right corner of $\mathbf{f}-\mathbf{j}$ represent the number of zebrafish embryos shown positive phenotypes versus the total number of embryos examined. Scale bar: $100 \mu \mathrm{m}$. Means \pm SEM are shown for three independent experiments. " $P<0.05$ (Student's $t$ test).

\section{Pdcd11-positive cells yield Tgf $\beta 1$ required for microglia differentiation}

Microglia differentiation requires step-wise-activated intrinsic transcription factors and circumstance-derived inducing factors, like Tgf $\beta 1$. Through comparing the RNA-seq data carried between $22 \mathrm{hpf}$ WT and pdcdll mutants, we noticed that tgfbla mRNA, a homolog of human $T G F B 1$, was profoundly reduced in $22 \mathrm{hpf} p d c d 11$ mutant embryos (Table S1). The finding was verified by qPCR and western blot (Fig. 2a, b). Using WISH, we found that $t g f b 1 a$ was highly expressed in cells on the YS at 22 hpf, at a location similar to Pdcd11, and that the expression of $t g f b l a$ was obviously reduced with $p d c d 11$ deficiency (Fig. 2c). Otherwise the expression of tgfblb, another orthologue of human TGFB1 were lowly expressed at 22 hpf and no difference was detected with $p d c d 11$ depletion
(Fig. S2i, j). Moreover, specially enriched Tgf $\beta 1$ in Pdcd 11 expression cells was revealed using an immunofluorescence assay (Fig. 2d). Tgf $\beta 1$-induced signature genes in mouse microglia have been studied [25, 26]. Supporting the role of pdcd11 in mediating Tgf $\beta 1$ activation in microglia, brain macrophages sorted from $p d c d 11$-deficient $\operatorname{Tg}($ mpeg $1: G F P)$ line showed reduced expression of Tgf $\beta 1$-stimulated genes, including sallla, tgfbrla and tgfbrlb, and increased expression of Tgf $\beta 1$-suppressed genes like tlr $5 a$ and ctsl.1 as compared with that in WT embryos (Fig. 2e). The elevation of $c s f l b$ and reduction of $c t s b a$ in microglia were also verified by WISH (Fig. 2f). Confirming the importance of $t g f b l a$ reduction in contributing to microglia depletion in $p d c d 11$ mutants, tgfbla overexpression restored both precursor and mature microglia numbers in pdcdll mutants (Fig. 2g-i). However, this rescue was compromised by the simultaneous repression of $\operatorname{Tgf} \beta$ activation in macrophages 
A

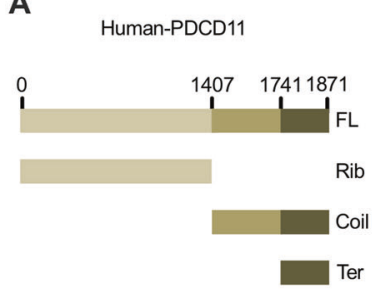

B

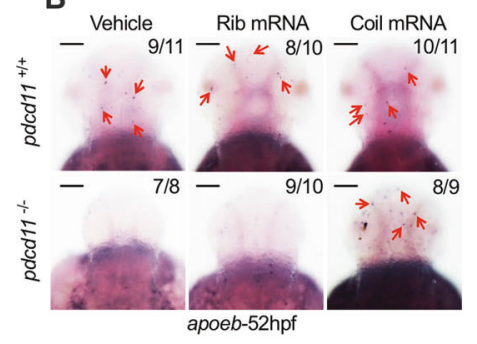

G
D

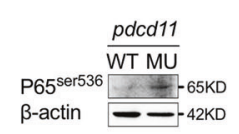

E

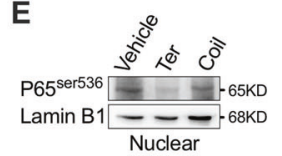

I

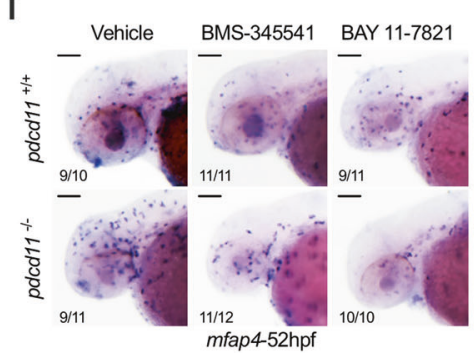

F

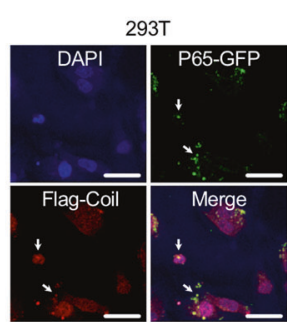

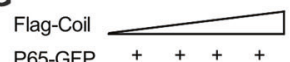

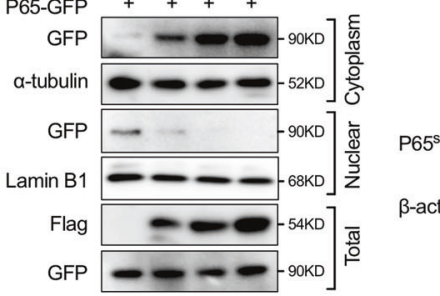

J

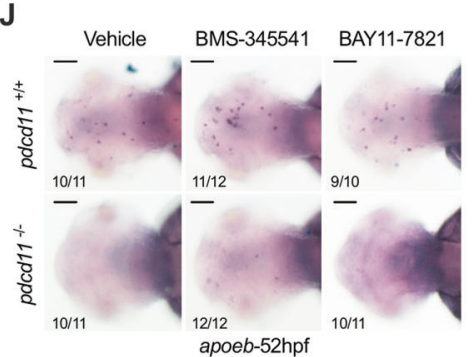

C

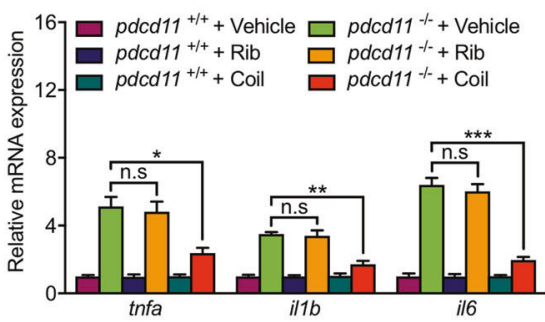

H

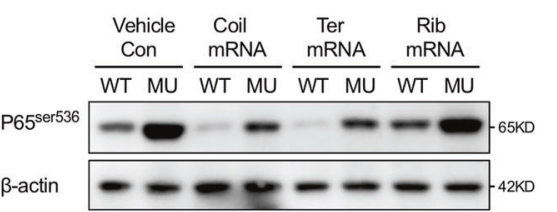

K

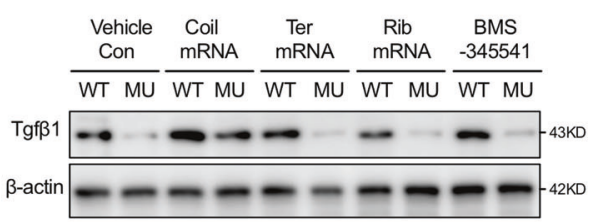

Fig. 3 P65 hyperactivation in pdcd11 mutants. a Schematic diagram showing different domains of PDCD11 consisting of Ribosome (Rib, 1-1407 aa), Coil (1408-1871 aa), and Terminal (Ter, 1742-1871 aa) domains. b Rescuing effects on apoeb positive microglia of pdcd11 mutants with the Rib or Coil domain mRNA overexpression. Red arrows indicate the apoeb positive cells. Scale bar: $100 \mu \mathrm{m}$. c qPCR assessment of inflammatory genes expression in pdcd11 mutants with Rib or Coil domain of PDCD11 overexpression. d Western blot examination of the 536-serine modified P65 expression in $22 \mathrm{hpf}$ WT and pdcd11 mutants. e Western blot examination of nuclear P65 536 contents with the Coil or Ter overexpression in HEK293T cells. f Immunofluorescence assay showing the expression pattern of cells co-transfected with N-terminal Flag fused Coil and P65-GFP. White arrowheads indicate the colocalized Coil and P65 in the cytoplasm or nucleolus. Scale bar: $20 \mu \mathrm{m}$. g Representative image of three independent experiments showing the effect of increasing Coil transfection on nuclear/cytoplasm P65 content. h Representative image of three independent experiments showing P65 ${ }^{536}$ levels in WT and pdcd11 mutants with Coil domain (Coil), Terminal domain (Ter), or Ribosome domain (Rib) mRNA overexpression. i Appearance of mfap4 expressing macrophages in pdcd11 mutants treated with $\mathrm{NF}-\kappa \mathrm{B}$ inhibitor.

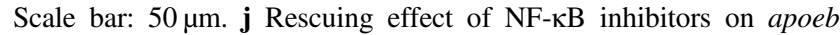
positive microglia in pdcd11 mutants. Scale bar: $100 \mu \mathrm{m}$. k Representative image of three independent experiments showing the rescue effects of Tgf $\beta 1$ levels with Coil domain (Coil), Terminal domain (Ter), Ribosome domain (Rib) mRNA or NF- $\kappa \mathrm{B}$ inhibitor addition in $22 \mathrm{hpf}$ pdcd11 mutants. The number positioned in the upper right corner of $\mathbf{b}$ and lower right corner of $\mathbf{i}, \mathbf{j}$ represent the number of zebrafish embryos shown positive phenotypes versus the total number of embryos examined. Means \pm SEM are shown for three independent experiments. n.s not significant, ${ }^{*} P<0.05 ;{ }^{* *} P<0.01 ;{ }^{* * *} P<0.001$ (Student's $t$ test). by either LY364947, a TGF $\beta$ R-I inhibitor addition, or overexpressing inhibitory cofactor smad7 under control of the mpegl promoter, implying the importance of Pdcd11derived $\operatorname{Tgf} \beta$ in regulating macrophage development (Fig. 2h-j).

\section{Elevated P65 transcriptional activity in pdcd11 mutants}

Aside from the large N-terminal ribosome domain (Rib), Pdcd11 contains C-Terminal Coil domain (Coil), the function of which has not been defined (Fig. 3a). To investigate the molecular mechanism, we first overexpressed Rib and Coil. While Rib overexpression faithfully restored P53 pathway genes, the disturbances of macrophage generation and inflammation pathway activation could only be reversed with Coil overexpression in pdcdl1 mutants (Fig. 3b, c and S3a-c). A short C-Terminal fragment (Ter) reportedly could interact with $\mathrm{P} 65$ (Fig. 3a), one member of

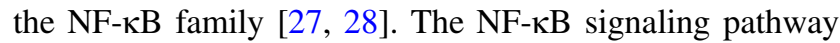
participates in modulating tissue immune homeostasis. Under normal circumstances, P65 is retained in the cytoplasm by the inhibitory protein IkappB and is available to the proteasome degradation pathway, which avoids 
A

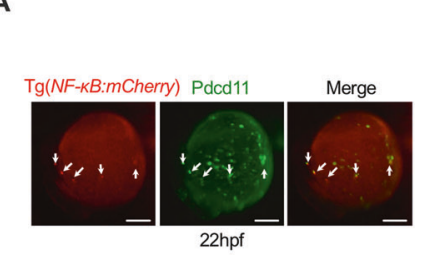

B

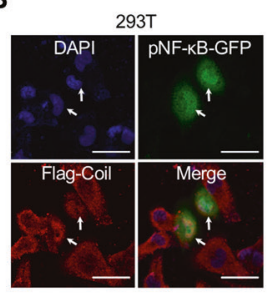

G c

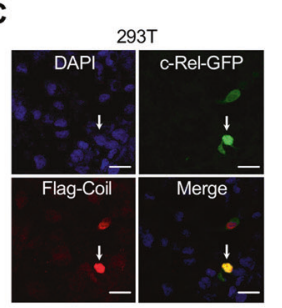

E

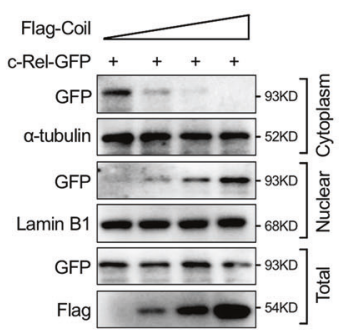

$\mathbf{F}$

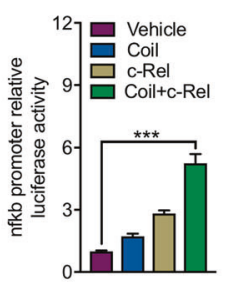

Contrista

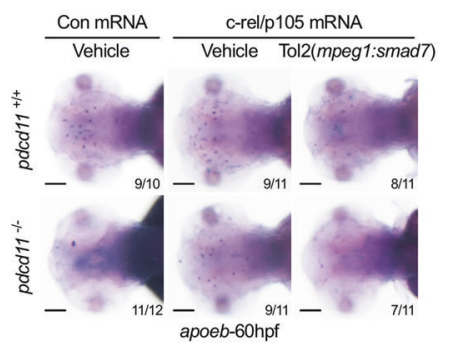

D

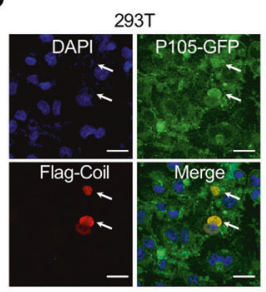

H

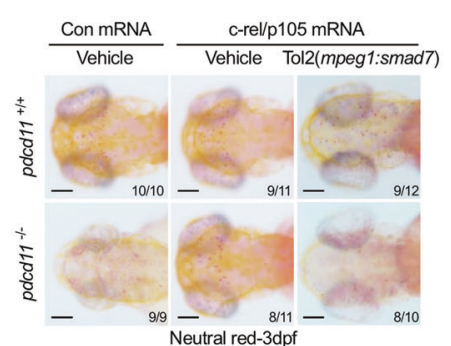

I

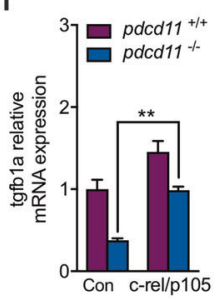

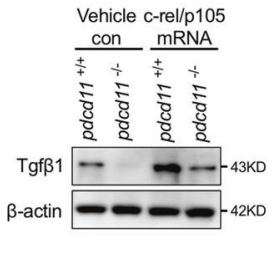

K

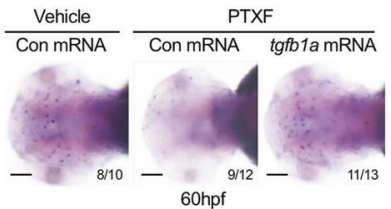

$\mathbf{L}$

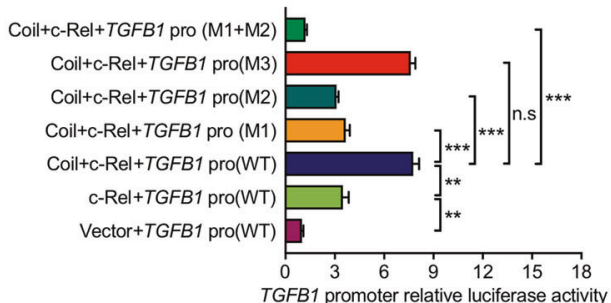

Fig. 4 PDCD11 promotes c-Rel mediated NF-אB activation. a IF assay of Pdcd11 expression in $\operatorname{Tg}(N F-\kappa B: m$ Cherry $)$ transgenic line. White arrowheads indicate the colocalized cells on the YS at $22 \mathrm{hpf}$. Scale bar: $100 \mu \mathrm{m}$. b In vitro transfection of plasmid containing NF-KB recognition motif driven GFP and Flag-Coil. White arrows indicate that cells with nuclear Coil expression displayed NF- $\mathrm{BB}$ activation. Scale bar: $20 \mu \mathrm{m}$. Representative image show cells co-transfected with Flag-Coil and c-Rel (c) or P105 (d). White arrows indicate that cells with nuclear expressed Coil and c-Rel (c) or P105 (d). Scale bar: 20 $\mu \mathrm{m}$. e. Western blot showing the effect of increasing Coil transfection on nuclear/cytoplasm c-Rel content. f Influence of Coil and c-Rel transfection on luciferase activities driven by the NF- $\mathrm{\kappa B}$-binding site. Microglia numbers in WT and pdcd11 mutants examined by apoeb (g) or Neutral Red staining (h) with c-rel and p105 mRNA or combined mpeg1 promoter driven smad7 plasmid injection. Scale bar: $100 \mu \mathrm{m}$. qPCR (i) and western blot (j) assessment of tgfbla or Tgf $\beta 1$ level in

uncontrolled inflammation activation. Upon stimulation, P65 is post-translationally modified by phosphorylation at the 536-serine residue, which obviates the interaction with IкB. P65 can then translocate into the nucleus to activate the downstream signaling pathway. We found that 536-serine phosphorylated P65 was obviously increased in pdcd11 mutants (Fig. 3d). In vitro overexpression of Coil or simply the terminal fragment Ter efficiently reduced nuclear 536serine phosphorylated P65 (Fig. 3e). Interestingly, we found that in vitro transfected P65 and Coil mostly colocalized in the cytoplasm or in the nucleolus, which correlated with reduced P65 transcriptional activity [29, 30] (Fig. 3f). The cytoplasm and nuclear P65 content with Coil overexpression were then examined respectively by western
$22 \mathrm{hpf}$ WT and pdcd11 mutants with $c$-Rel and p105 mRNA overexpression. k Microglia numbers in embryos with c-Rel inhibition (PTXF) or combined tgfbla mRNA complementation. Scale bar: 100 $\mu \mathrm{m}$. I Reporter assay showing the regulation of $T G F B 1$ promoter by Coil and c-Rel transfection. TGFB1 WT promoter containing three cRel-binding sites (5'-ccGGGGcaccccc- $3^{\prime}, \quad-761 \mathrm{bp}$ to TSS; 5'ggGGGGacgecccgt- $3^{\prime}, \quad-776$ bp to TSS; $5^{\prime}$-aaGGGAcccccctcg- $3^{\prime}$, $-1019 \mathrm{bp}$ to TSS). The three binding motifs were respectively mutated and designated as M1 (5'-ccAAAAcaccccc- $3^{\prime},-761 \mathrm{bp}$ to TSS), M2 (5'-ggAAAAacgeccegt- $3^{\prime}, \quad-776$ bp to TSS), M3 (5'aaAAACccccctcg- $3^{\prime},-1019 \mathrm{bp}$ to TSS). The number positioned in the lower right corner of $\mathbf{g}, \mathbf{h}, \mathbf{k}$ represents the number of zebrafish embryos shown positive phenotypes versus the total number of embryos examined. Means \pm SEM are shown for three independent experiments. n.s not significant, ${ }^{*} P<0.05 ;{ }^{* *} P<0.01 ;{ }^{* * *} P<0.001$ (Student's $t$ test).

blot. Increasing Coil transfection obviously increased the P65 cytoplasm content, while correspondingly reducing the nuclear P65 level (Fig. 3g). Moreover, increased 536serine phosphorylated P65 in the pdcd11 mutants were restored by either Coil or Ter domain overexpression, but not by Rib domain overexpression (Fig. 3h). To determine whether the rescuing effects of Coil relied on its capacity in suppressing P65 activation, two NF- $\mathrm{kB}$ inhibitors, BMS345541 and BAY 11-7821, were used [31]. Treatment with either BMS-345541 or BAY 11-7821 reduced 536-serine phosphorylated $\mathrm{P} 65$, and inflammatory genes and macrophages also returned to normal in pdcdll mutants (Fig. 3i and S3d, e). A correctly regulated inflammasome cascade is required for the maintenance of microglia homeostasis [32]. 
However, microglia number or the TGF $\beta 1$ level could not be rescued by BMS-345541 or BAY 11-7821 treatment (Fig. 3j, k), suggesting that other P65 independent mechanisms might be utilized by Coil in regulating microglia generation.

Interestingly, while Coil overexpression restored both macrophage appearance and inflammatory genes in the pdcd11 mutants, similar efficacy was not detected with either Coil overexpression in pan neuron cells under Huc promoter (also named embryonic lethal, abnormal visionlike, neuron-specific RNA-binding protein 3, elavl3) [33] or macrophages under control of the mpegl promoter [34] (Fig. S3c, f).

\section{Pdcd11 enhances c-Rel/p105 nuclear transport}

Transgenic zebrafish with NF- $\kappa \mathrm{B}$ recognition sequences driven fluorescent protein was utilized to study NF- $\kappa B$ activity in zebrafish [35]. Helped by this transgenic line, we were surprised to find that although PDCD11 suppressed $\mathrm{P} 65$ activation, NF- $\mathrm{kB}$ was otherwise activated in Pdcd11expressing cells on the YS at $22 \mathrm{hpf}$ (Fig. 4a). In comparison with cytoplasm Coil expressed cells, cells with nuclear Coil expression were prone to activate EGFP under control of the NF- $\mathrm{KB}$ recognition sequences (Fig. $4 \mathrm{~b}$ and S4a).
Among the five members of NF-кB family, P65, c-Rel, and Rel-B harbor the transcription activation domain (TAD), which binds NF- $\mathrm{KB}$ recognition sequences and activates the expression of downstream genes [36, 37]. The findings prompted us to speculate that this activation of the NF- $\mathrm{kB}$ binding element relied on the transcriptional activity of other members of the NF- $\mathrm{kB}$ family. Indeed, different from P65, Coil colocalized with c-Rel and P105 in the nucleus and Coil overexpression promoted the nuclear retention of c-Rel (Fig. 4c-e and S4b, c). A luciferase assay showed that Coil co-expression enhanced the capacity of c-Rel in activating the NF-KB-binding element (Fig. 4f). Confirming the importance of reduced nuclear c-Rel/P105 in leading the microglia deficiency in $p d c d 11$ mutants, we found that cRel and p105 co-overexpression recovered microglia numbers as manifested by either apoeb or Neutral Red staining (Fig. 4g, h). These rescue effects were largely compromised when repressive smad7 was co-expressed in macrophages, suggesting that c-Rel/P105 functions upstream of TGF $\beta$ signaling (Fig. 4g, h). Consistently, the expression level of Tgf $\beta 1$ was also increased with c-Rel and p105 mRNA overexpression (Fig. 4i, j). Injection of the xanthine derivative pentoxifylline (PTXF), which selectively degrades cRel [38], also reduced the number of microglia in WT embryos. The reduction could also be recovered by the
A

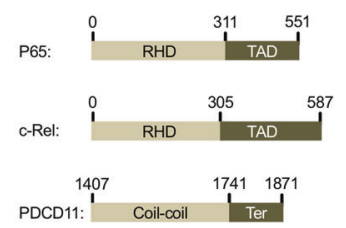

$\mathrm{F}$

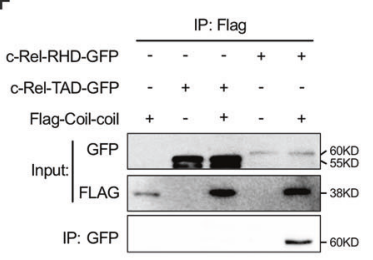

B

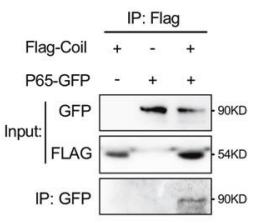

G

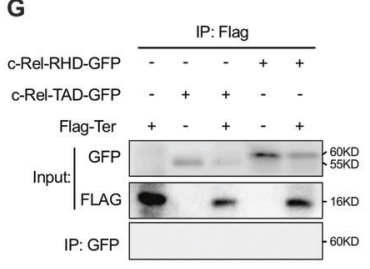

Fig. 5 Different interaction pattern between P65 or c-Rel and PDCD11. a Schematic diagram showing the domain structures of human P65, c-Rel, and PDCD11-Coil. b Immunoprecipitation (IP) assay of the interaction between P65-GFP and Flag-Coil. c IP assay of the interaction between c-Rel-GFP and Flag-Coil. d IP assay of the interaction between the Coil-coil fragment of PDCD11 with the RHD domain or TAD domain of P65. e IP assay of the interaction between the terminal (Ter) fragment of PDCD11 with the RHD domain or TAD domain of P65. f IP assay of the interaction between the Coil-coil fragment of PDCD11 with the RHD domain or TAD domain of c-Rel. g IP assay of the interaction between the terminal (Ter) fragment of PDCD11 with the RHD domain or TAD domain of c-Rel. $\mathbf{h}$ Western blot examination of the influential role of overexpression Coil, Coil-coil and Terminal of PDCD11 on P65 $5^{\text {ser536 }}$ expression in $22 \mathrm{hpf}$ pdcd11 mutants. i Western bot show the effect of increasing Ter
D

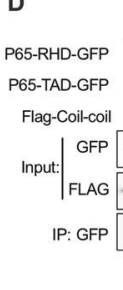

H

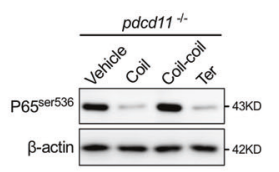

E

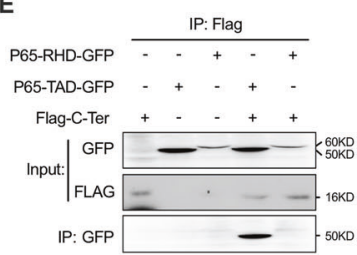

J

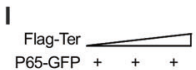
P65-GFP +++

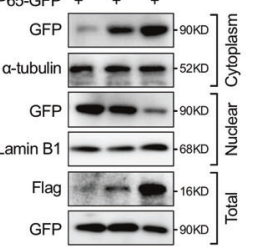

J

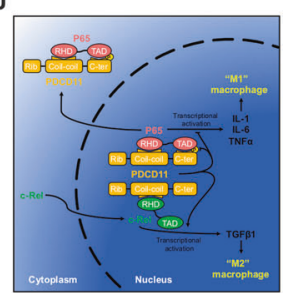

transfection on nuclear/cytoplasm P65 content in 293T cells. j Schematic diagram showing the working model for PDCD11-mediated regulation of c-Rel and P65. Under normal circumstances, on one hand, the Ter fragment of PDCD11 (yellow) binds and masks the phosphorylation site of P65 (pink), which is required for its nuclear translocation and transcriptional activation; on the other hand, the only existing interaction between the coil-coil of PDCD11 and the RHD domain of c-Rel contrarily promotes c-Rel (green) nuclear retention for activating TGF $\beta 1$ expression, which drives macrophage differentiation toward microglia with "M2" properties. However, cells deficient in PDCD11 show increased P65 nuclear translocation, which tends to lead to activation of inflammation-related genes, such as $I L-1, I L-6$, and $T N F \alpha$. These cytokines then facilitate "M1" macrophage differentiation. 
A

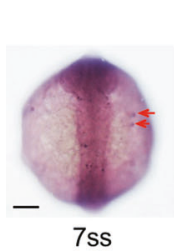
pdcd11-WISH

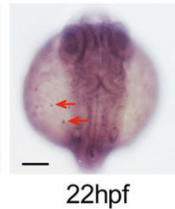

B
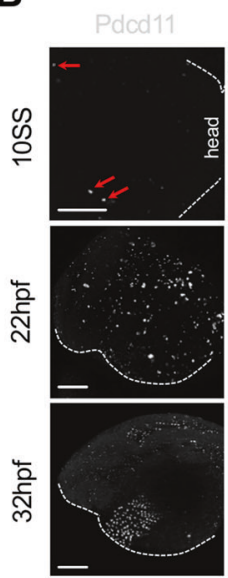

$\operatorname{Tg}($ pu. 1:GFP $)$
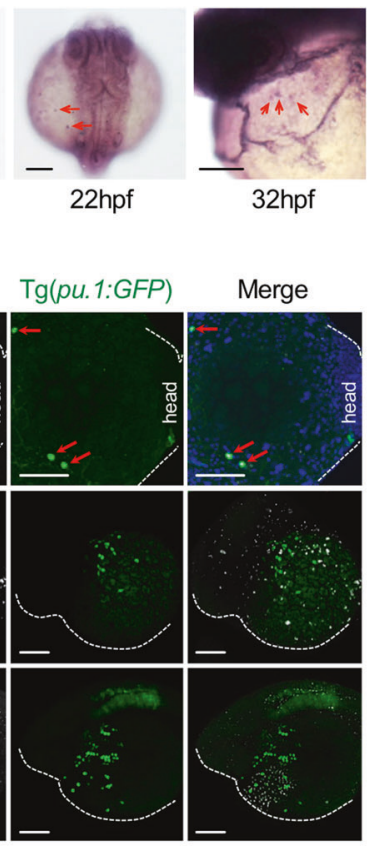

C

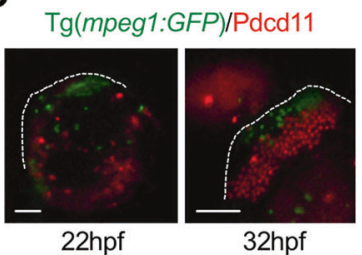

D

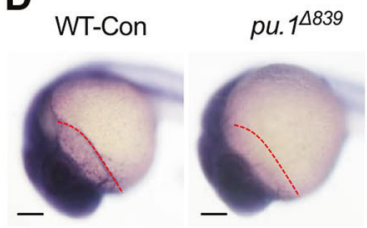

$32 \mathrm{hpf}$

$\mathbf{E}$

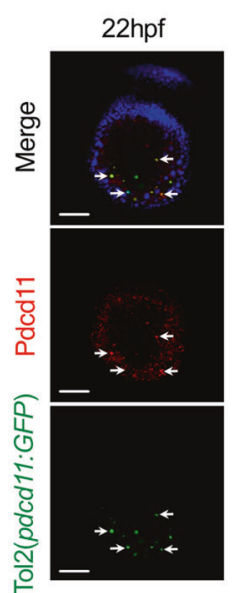

$\mathbf{F}$

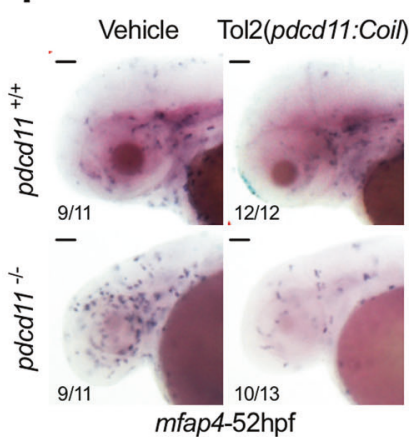

G

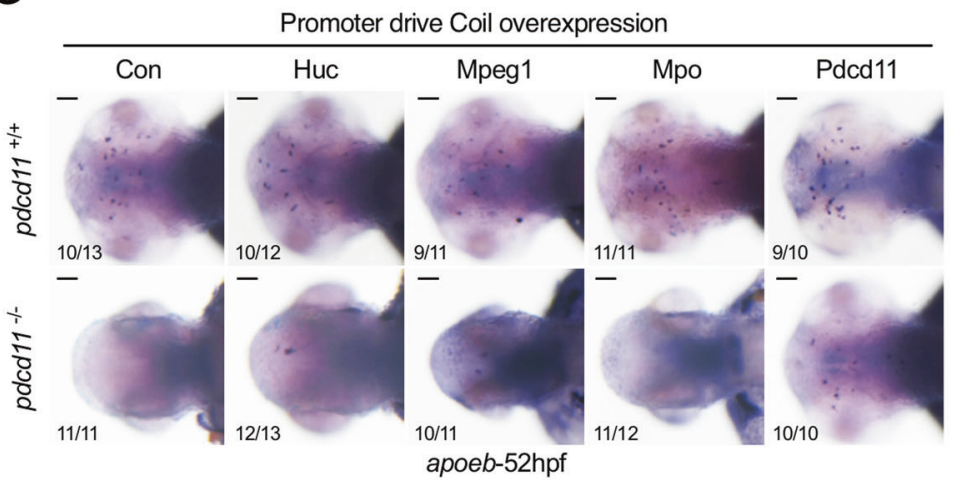

Fig. 6 Pdcd11 expression pattern examined during zebrafish embryonic development. a WISH assessment of pdcd11 expression in zebrafish at 7-ss, $22 \mathrm{hpf}$, and 32; hpf. The red arrows indicate pdcd11 signals on the YS and pericardial cavity. b Immunofluorescence staining of Pdcd11 in $\operatorname{Tg}($ pu.1:GFP) zebrafish. The red arrows indicate colocalized Pdcd11 and Pu.1 expressing cells on the YS at 10-ss. c Pdcd11 expression in $22 \mathrm{hpf}$ and $32 \mathrm{hpf} \operatorname{Tg}($ mpeg $1: G F P)$ zebrafish assayed by immunofluorescence staining. d WISH examination of pdcd11 expression in pu.1 deficient embryos. The red dotted lines denote the pericardial cavity underneath the brain where pdcd11

injection of tgfbla mRNA (Fig. 4k). In a previous study, chromatin immunoprecipitation combined with microarray technology (ChIP/Chip) examination of mitogen-stimulated $\mathrm{T}$ cells Rel/NF-kappa-B revealed potential Rel/NF- $\mathrm{KB}$ recognition motifs in the promoter region of TGFB1 [39]. To further determine the regulatory role on TGFBI by Coil and c-Rel, HEK293 cells were examined using a luciferase reporter assay. Coil co-expression enhanced the capacity of c-Rel in stimulating the TGFBI promoter. This expressed. e IF assessment of the co-expression between GFP driven by $p d c d 11$ promoter and endogenous Pdcd11. White arrowheads indicate the colocalization signal on the $22 \mathrm{hpf}$ YS. f The expression pattern of mfap4-positive macrophages in the $52 \mathrm{hpf}$ brain with pdcd11 promoter driven Coil overexpression. g Rescue effects of apoeb positive microglia with Coil overexpressed under different promoters including Huc, mpeg1, mpo, and pdcd11. The number positioned in the lower left corner of Fig. 6f, g represent the number of zebrafish embryos shown positive phenotypes versus the total number of embryos examined. Scale bar: $100 \mu \mathrm{m}$.

enhancement was abolished by the combined M1 and M2 cRel-binding site mutation (Fig. 41). These results suggest that Coil and c-Rel promotes tgfbl expression in early zebrafish embryos.

Since P65 and c-Rel are structurally similar and both harbor an N-terminal Rel homology domain (RHD) and CTerminal TAD domain, it was of interest to investigate how PDCD11 distinguished the two (Fig. 5a). Co-IP examination revealed that both $\mathrm{P} 65$ and c-Rel could interact with the 
Coil domain of PDCD11 (Fig. 5b, c). The Coil domain was further divided into the Coil-coil and Ter fragment, which displayed a different interaction pattern with P65 and c-Rel. Since the RHD domain of P65 and c-Rel interact with the Coil-coil region of PDCD11, the Ter fragment failed to bind to the TAD domain of c-Rel as observed with P65 (Fig. $5 \mathrm{~d}-\mathrm{g}$ ). This result was reasonable since, because the RHD domains of NF- $\mathrm{KB}$ family members are similar, their TAD domains are much more distinct. The interaction of Ter with the TAD domain of P65 might cover the phosphorylation site required for P65 transcriptional activation. The mere overexpression the Ter fragment could suppress the P65 activation in pdcd11 mutants, otherwise the Coil-coil fragment overexpression failed to do so (Fig. 5h). Confirming the importance of Ter fragment in inhibiting P65 activation, the overexpression of this fragment also inhibited P65 nuclear translocation (Fig. 5i). Thus, the differential interactions between the TAD domains and PDCD11 might contribute to the different regulation mode of P65 and c-Rel by PDCD11 (Fig. 5j).

\section{YS Pdcd11-expressing cells are derived from Pu.1- progenitors}

By WISH, we found that aside from the obvious expression signal in the neuron epithelium, scattered cells on the YS were also detected from 7-somite stages (ss) (Fig. 6a). At $32 \mathrm{hpf}, p d c d 11$-positive cells had accumulated in the pericardial cavity, the site of inflow of all blood cells (Fig. 6a). In zebrafish, myeloid progenitor cells expressing Pu.1 emerged within the anterior lateral plate mesoderm (ALPM) at the 5-ss and then migrated into the rostral blood island (RBI) and onto the YS. This was followed by differentiation of the cells into macrophages and neutrophils, which then seeded the brain from approximately $24 \mathrm{hpf}$. Through use of the zebrafish $\operatorname{Tg}(P u .1: G F P)$ transgenic line, we found that at 10 -ss in the ALPM region, Pdcd11 was activated in a subset of Pu.1-GFP-positive cells (Fig. 6b). However, this co-expression pattern reduced at $22 \mathrm{hpf}$ and thereafter remained absent, suggesting later silencing Pu.1 expression (Fig. 6b). The expression of Pdcd11 in macrophages and neutrophils was also examined using $\operatorname{Tg}($ mpeg $1: G F P)$ and $\operatorname{Tg}($ mpo:GFP) transgenic lines, respectively. Mpeg1positive macrophages appeared to be closely surrounded by Pdcd11-positive cells in the pericardial cavity underneath the brain at $32 \mathrm{hpf}$, but no colocalizations were detected (Fig. 6c). Similarly, no colocalizations between Pdcd11 and mpo-positive neutrophils were detected (Fig. S4d). Intriguingly, in pu.1-deficient embryos, while pdcdl1 expression in the neuron epithelium remained unchanged, its expression in the pericardial cavity was disappeared, implying that the scattered $p d c d 11$-expressing cells observed on the YS were Pu.1-dependent (Fig. 6d). To verify the $p d c d 11$ expression pattern, the $1.86 \mathrm{~kb}$ genomic region upstream of the translation initiation site of zebrafish pdcdl1 was cloned into a transposon vector containing EGFP. The pdcdl1 promoter-driven GFP-expressing cells on the YS strongly colocalized with endogenous Pdcd11 at $24 \mathrm{hpf}$ in an immunofluorescence assay (Fig. 6e). Overexpression of Coil under the control of the $1.86 \mathrm{~kb} p d c d 11$ promoter reversed the macrophage imbalance in $p d c d 11$ mutants, with the number of inflammatory macrophages reduced and that of microglia increased (Fig. 6f, g). This rescue effect was not observed in embryos overexpressing Coil overexpression under the control of neuron (Huc), macrophage (Mpeg1), or neutrophil (Mpo) specific promoters (Fig. 6g). Collectively, these results suggest that scattered Pdcd11-expressing cells may be derivatives of Pu.1-positive progenitors, but that they are distinct from previously identified neutrophils or macrophages.

\section{Discussion}

WMH examined by magnetic resonance imaging have been used to describe WM changes related with cognitive dysfunction, stroke injury, cerebral small vessel disease, and neurodegenerative disorders [40]. Key cellular components of the gliovascular unit including astrocytes, oligodendrocytes, pericytes and microglia. It was suggested that microglia within the WM be associated functions like surveillance at the blood-brain barrier and clearance debris $[41,42]$. In this study, helped by zebrafish model organism, we found that deficiency of $p d c d 11$, a WMH susceptibility gene, led to completely lost of mature microglia in the brain. In mouse, helped by in vivo lineage tracing, it was found that adult microglia derive from primitive myeloid progenitors that arise before embryonic day 8 [43]. However, as with zebrafish, multiple waves of microglia exist and originate from distinct hematopoietic precursors were identified [44]. Despite the different ontogeny of primitive and adult microglia between mouse and zebrafish identified, the regulators underlying the differentiation of EMP progenitors toward microglia remain undefined. TGF- $\beta$ family members are cytokine required for the maintenance of the microglia-specific homeostatic gene signature. Deletion of the TGF- $\beta$ receptor in microglia resulted in rapid conversion of microglia toward an inflammatory macrophage phenotype $[15,16]$. Mice harboring mutated TGFB1 exhibit an acute wasting syndrome followed by death [45]. The most prominent lesions are tissue necrosis in specific organs and multifocal, mixed inflammatory cell infiltration into numerous organs [46]. Despite accumulating evidence for local expression of TGF- $\beta 1$ in the nervous system during inflammation, the cellular source of TGF- $\beta 1$, which drives microglia differentiation is unknown. Pdcdll-deficient 
embryos exhibit brain necrosis and organ inflammatory cell infiltration, which is very similar to what is observed with TGFB1-mutated mice. Moreover, Pdcd11-expressing cells on the YS colocalized with TGF- $\beta 1$-positive cells at $22 \mathrm{hpf}$ on the YS. Both the mRNA and protein levels of TGF- $\beta 1$ were significantly reduced with $p d c d 11$ depletion. Importantly, overexpression of TGF- $\beta 1$ under the control of the pdcd11 promoter faithfully recovered the microglia numbers in pdcd11 mutants. Thus, our observations support the notion that during the early development in zebrafish, Pdcd11-expressing cells might be one of the major source of TGF- $\beta 1$ to induce microglia maturation.

In studying the property of YS Pdcd11-expressing cells, we found that these cells are distinct from initially identified macrophages and neutrophils, but they are also derived from $\mathrm{Pu}$.1-expressing progenitors and require Pu.1. The NF- $\mathrm{kB}$ family member possesses distinct biological functions and controls the expression of different target genes, manifesting as unique developmental phenotypes between NF-kB/Rel family member knock-out mice. The distinct and opposing functions of P65 and cRel in regulating cellular functions, such as proliferation and apoptosis, have been revealed in several different models [47, 48]. In P105 and c-Rel double knock-out mice, while peripheral T-cell populations developed normally, follicular, marginal zone, and $\mathrm{CD}^{+}$peritoneal B-cell populations were all reduced, suggesting the particular requirement of P105 and c-Rel in these cell types [49]. It was reported that B1a cells are abundant in the neonatal mouse brain and promote oligodendrogenesis by regulating the proliferation of oligodendrocyte-precursor cells and the number of microglia [50]. As the definitive markers for B1a lymphocytes in zebrafish are elusive, other model organism like mice might be useful for our understanding Pdcd11-positive cells in the future. A recent report in studying the mpeg 1.1:GFP reporter line, it found that beside macrophages, a subpopulation of Blymphocytes is marked by mpegl.1 reporters in most adult zebrafish organs, with highly expressed $c d 79 a$, $i g h m$, and ighd [51]. This would also be helpful for our future examination of this transgenic line in study the property of Pdcd11-positive cells.

\section{Materials and methods}

\section{Experimental model}

Zebrafish maintenance and staging were performed as previously described [52]. The zebrafish facility and study were approved by the Institutional Review Board of the Institute of Health Sciences, Shanghai Institutes of Biological Sciences, Chinese Academy of Sciences (Shanghai,
China), and the methods were carried out in accordance with the approved guidelines. The $T g(p u .1:$ GFP) [53], $T g$ (mpeg 1: GFP) [54], Tg(mpo: GFP) [55], P53 $3^{\mathrm{zd1} 1 / z \mathrm{df} 1}$ mutants $[20,56], p u .1^{\Delta 839}$ zebrafish lines were used.

\section{Pdcd11 knock-out zebrafish line generation}

For CRISPR-Cas9 mediated pdcdl1 knock-out zebrafish generation, guide RNA (gRNA) targeting exon4 of $p d c d 11$ (5'-GGTCTCCCGAGCGGCCTCGT-3') was designed using the online tool, ZiFiT Targeter software (http://zifit.pa rtners.org/ZiFiT), and was synthesized by cloning the annealed oligonucleotides into the sgRNA expression vector as previously described [57]. The mutant line is screened by Bsrbi enzyme (NEB) cutting and confirmed by Sanger sequencing.

\section{mRNA synthesis, and microinjection}

mMACHINE Kit (Ambion) was used for mRNA transcription. For promoter-driven Coil or smad7 expression in zebrafish, $30 \mathrm{ng} / \mu \mathrm{l}$ transposase mRNA was coinjected.

\section{Chemical treatment}

PTXF (Abcam) was injected (0.5 ng) into one-cell stage embryos. BMS-345541 (Selleckchem, $10 \mu \mathrm{M}$ ), BAY 117821 (Selleckchem,10 $\mu$ M), and LY364947 (Selleckchem, $10 \mu \mathrm{M}$ ) were used for treating embryos from $18 \mathrm{hpf}$.

\section{Plasmids}

The Tol2(NFKB:EGFP) plasmid was obtained from Addgene [35]. The $1.86-\mathrm{kb}$ sequence immediately proximal to the $p d c d 115^{\prime}$ untranslated region was amplified and cloned into a modified pT2cfosGW vector containing the zebrafish Tol2 transposable element [35]. The resulting construct was injected, together with transposase mRNA, into one-cell stage WT TU embryos [58]. The full length PDCD11 was cloned into vector pcDNA3.1. The C-coil, C-coil-coil, and C-Ter domain of human PDCD11 were amplified and cloned into vector $\mathrm{pCS} 2^{+}$. Full-length human c-Rel, P65, and P105 were cloned into $\mathrm{pCS}^{+}$with GFP fused in the C-terminus. The RHD and TAD domain of c-Rel and P65 were amplified from the full-length vector and cloned into $\mathrm{pCS}^{+}$. Full-length zebrafish tgfbla was amplified from zebrafish cDNA and cloned into the $\mathrm{pCS}^{+}$vector. Mpeg1 promoter driven C-coil and smad7 were cloned by replacing dendra in vector Tol2 (mpeg1:dendra2) (Addgene, 51462). Tol2(mpx:Coil) was cloned by replacing the Lifeact-Ruby (Cla1 and Sal1) with Coil in Tol2(mpx:Lifeact-Ruby) (Addgene, 45246). Tol2(huc: Coil) was also obtained by gateway cloning using p5E-Huc (Addgene, 72640). 
WISH

WISH was performed as previously described [59]. Probes for l-plastin, csflra, and mpo were used as previously described [10, 60, 61]. Antisense probes, including il6, illb, mfap4, apoeb, pdcd11, csfla, ctsba, tgfbla, tgfblb, cxcr3.1, and $c x c r 3.2$ were transcribed in vitro from PCR products amplified from zebrafish cDNA with the T7 transcriptase binding sequence added to the reverse primers. Primers used are shown in Table S2.

\section{Neutral Red, AO, TUNEL, pH3, and immunofluorescence assay}

For Neutral Red staining, zebrafish larvae collected at 60 hpf and $3 \mathrm{dpf}$ were soaked in Neutral Red (Sigma-Aldrich, $\mathrm{N} 6264 ; 2.5 \mathrm{mg} / \mathrm{mL}$ ) overnight at $28.5^{\circ} \mathrm{C}$. For AO staining, embryos recovered at $22 \mathrm{hpf}$ were incubated in $10 \mu \mathrm{g} / \mathrm{mL}$ AO stain (Sigma-Aldrich) dissolved in E3 medium in the dark for $\sim 30 \mathrm{~min}$. TUNEL staining was performed as previously described [62]. $\mathrm{pH} 3$ and immunofluorescence staining were carried out using the following antibodies: PDCD11 (Sigma-Aldrich; HPA017924; 1:100); pH3 (Santa Cruz Biotechnology; 1:500), TGF- $\beta 1$ (Sigma-Aldrich, T0438; $25 \mu \mathrm{g} / \mathrm{mL})$

\section{qPCR analysis}

After treatment with DNAaseI (Ambion), RNA was prepared using Trizol reagent (Invitrogen, H10522) and then subjected to cDNA synthesis using a cDNA synthesis kit (ABI). Real-time PCR was performed using a Fast Start Universal SYBR ${ }^{\circ}$ Green Master Rox probe (Roche Applied Science, 13800300) and Mastercycler thermal cycler (Eppendorf, 22331). Primers are summarized in Table S2.

\section{Western blot, nuclear extraction, and co-IP}

Zebrafish embryos at the indicated developmental stages were de-yolked and homogenized in lysis buffer mixed with proteinase inhibitor as previously described [63]. Nuclear proteins were extracted by the NE-PER ${ }^{\mathrm{TM}}$ Nuclear and Cytoplasmic Extraction Reagents (Thermo Fisher Scientific, 78833). For the co-IP assay, proteins were purified in RIPA buffer $(50 \mathrm{mM}$ Tris, $150 \mathrm{mM} \mathrm{NaCl}, 10 \%$ glycerol, $5 \mathrm{mM} \mathrm{MgCl}, 0.5 \% \mathrm{NP} 40$, and Roche cocktail protease inhibitor) $48 \mathrm{~h}$ after transfection, and mixed with anti-c-Flag agarose affinity gel antibody (Sigma-Aldrich, A220). After incubation at $4{ }^{\circ} \mathrm{C}$ overnight, beads were collected for western blotting. Samples were subjected to sodium dodecyl sulfate-polyacrylamide gel electrophoresis and immunoblotting according to standard protocols. The antibodies used were anti-GFP (SigmaAldrich, G1544), anti-Flag (Sigma-Aldrich, F7425), anti-
PDCD11 (Sigma-Aldrich; HPA017924), anti-P65 ${ }^{\text {ser536 }}$ (Cell Signaling Technology, 3031), anti- $\alpha$-Tubulin (Sigma-Aldrich, T9026), anti- $\beta$-Actin (Cell Signaling Technology, 8457), and anti-LaminB1 (Cell Signaling Technology, 13435).

\section{Cell culture and dual-luciferase reporter assay}

Cells were cultured in a humidified incubator at $37^{\circ} \mathrm{C}$ in the presence of $5 \% \mathrm{CO}_{2}$. 293T and Hela cells obtained from ATCC were cultured in DMEM medium supplemented with $10 \%$ fetal bovine serum, $100 \mathrm{IU} / \mathrm{mL}$ penicillin, and $100 \mu \mathrm{g} /$ $\mathrm{mL}$ streptomycin (Gibco). Identity of cell lines were validated by STR analysis. Mycoplasma testing was routinely performed on all cells used in the study, and confirmed to test negative. For cell transfection, Effectene transfection reagent (Q1AGEN, 1012829) was used according to the manufacturer's instructions. NF- $\mathrm{kB}$ binding motif driven luciferase reporter and human $T G F B 1$ (1.3-kb upstream the transcription start site) was used. C-Coil (150 ng) and/or c-Rel (150 ng) as well as Renilla constructs ( $5 \mathrm{ng}$ ) were transfected into 293 $\mathrm{T}$ cells (60\%-70\% confluence per well in 24-well plates) and luciferase activities were assessed according to the manufacturer's instructions (Promega). Site mutagenesis was carried on the TGFB1 promoter (Invitrogen, A13282)

\section{Cell sorting and cytology}

$\mathrm{Tg}$ (mpeg 1:EGFP) transgenic embryos at $60 \mathrm{hpf}$ were connected and digested with $0.5 \%$ trypsin (Gibco) for 15 min at $37{ }^{\circ} \mathrm{C}$ to analyze microglia gene signature. Sixty hours post fertilization zebrafish brain was cut before digestion. After centrifugation at $400 \times g$ for $5 \mathrm{~min}$, two washes with PBS, and passage through a $40 \mu$ m nylon mesh filter, the single cell suspension was subjected to fluorescence-activated cell sorting using a MoFlo device (DakoCytomation). EGFP positive cells were used for downstream qPCR assay.

\section{Cell IF assay}

Cell IF were carried as previously described. Cells grown to $60 \%$ confluence on coverslips were first fixed and blocked and then incubated with mouse anti-FLAG (Sigma-Aldrich, F7425). Secondary antibody goat anti-mouse, Alexa Fluor 594 (Invitrogen).

\section{Imaging}

Zebrafish IF stain images were taken using an Olympus FV1000 scanning confocal microscope. The confocal images were captured with an UPLSAPO $40 \times$ or $60 \times$ objective. Immuno-stained cell images were collected using a Leika with an UPLSAPO $60 \times$ objective. 


\section{RNA-sequencing (RNA-seq)}

Total RNA from $22 \mathrm{hpf}$ WT and pdcdl1 mutants was collected respectively and subjected to RNA-seq. Shanghai Novel Bioinformatics Company provided the deepsequencing service.

\section{Statistical analysis}

For all experiments carried, zebrafish embryos were randomized distributed to each group. The outcome of experiments were assessed according to phenotypic changes. Statistical analyses were conducted using the Student's $t$ test (two-sided) or log-rank tests. Statistical significance was taken to be $P<0.05$.

Acknowledgements We are grateful for Min Deng, Mei Dong, Yi Chen, Yi Jin, Zheng Ruan, Juan Chen, and Yan Zhao for their excellent technical support. This work was supported by grants from National Science Foundation of China (No. 81970098, 81803014 and 81802424), China Postdoctoral Science Foundation (No. 2019M651518).

Author contributions JZ and RY developed the concept of this study. $\mathrm{RY}$ and MZ performed most of the experiments in zebrafish and cell lines. RY, MZ, and MG contributed in vector construction and western blot. RY, and YW helped in cell culture, qPCR and the in situ assays. YZ, WZ provide the $p u .1^{\Delta 839}$ zebrafish lines. HY provided the $c e b$ $p a^{\Delta \mathrm{CA}}$ zebrafish line. JZ, RY, SZ, CZ HT, and JZ prepared the draft and final version of the manuscript. All authors reviewed the results and approved the manuscript.

\section{Compliance with ethical standards}

Conflict of interest The authors declare no competing financial interests.

Publisher's note Springer Nature remains neutral with regard to jurisdictional claims in published maps and institutional affiliations.

Open Access This article is licensed under a Creative Commons Attribution 4.0 International License, which permits use, sharing, adaptation, distribution and reproduction in any medium or format, as long as you give appropriate credit to the original author(s) and the source, provide a link to the Creative Commons license, and indicate if changes were made. The images or other third party material in this article are included in the article's Creative Commons license, unless indicated otherwise in a credit line to the material. If material is not included in the article's Creative Commons license and your intended use is not permitted by statutory regulation or exceeds the permitted use, you will need to obtain permission directly from the copyright holder. To view a copy of this license, visit http://creativecommons. org/licenses/by/4.0/.

\section{References}

1. Debette S, Markus HS. The clinical importance of white matter hyperintensities on brain magnetic resonance imaging: systematic review and meta-analysis. BMJ. 2010;341:c3666.
2. Fornage M, Chiang YA, O'Meara ES, Psaty BM, Reiner AP, Siscovick DS, et al. Biomarkers of inflammation and MRI-defined small vessel disease of the brain: the Cardiovascular Health Study. Stroke. 2008;39:1952-9.

3. Gouw AA, Seewann A, van der Flier WM, Barkhof F, Rozemuller AM, Scheltens P, et al. Heterogeneity of small vessel disease: a systematic review of MRI and histopathology correlations. J Neurol Neurosurg Psychiatry. 2011;82:126-35.

4. Wright CB, Moon Y, Paik MC, Brown TR, Rabbani L, Yoshita $\mathrm{M}$, et al. Inflammatory biomarkers of vascular risk as correlates of leukoariosis. Stroke. 2009;40:3466-71.

5. Fornage M, Debette S, Bis JC, Schmidt H, Ikram MA, Dufouil C, et al. Genome-wide association studies of cerebral white matter lesion burden: the CHARGE consortium. Ann Neurol. 2011;69:928-39.

6. Hofer E, Cavalieri M, Bis JC, DeCarli C, Fornage M, Sigurdsson $\mathrm{S}$, et al. White matter lesion progression: genome-wide search for genetic influences. Stroke. 2015;46:3048-57.

7. Verhaaren BF, Debette S, Bis JC, Smith JA, Ikram MK, Adams $\mathrm{HH}$, et al. Multiethnic genome-wide association study of cerebral white matter hyperintensities on MRI. Circ Cardiovasc Genet. 2015;8:398-409.

8. Lee J, Hamanaka G, Lo EH, Arai K. Heterogeneity of microglia and their differential roles in white matter pathology. CNS Neurosci Ther. 2019;25:1290-8.

9. Orihuela R, McPherson CA, Harry GJ. Microglial M1/M2 polarization and metabolic states. Br J Pharmacol. 2016;173:649-65.

10. Herbomel P, Thisse B, Thisse C. Zebrafish early macrophages colonize cephalic mesenchyme and developing brain, retina, and epidermis through a M-CSF receptor-dependent invasive process. Dev Biol. 2001;238:274-88.

11. Mazzolini J, Le Clerc S, Morisse G, Coulonges C, Kuil LE, van Ham TJ, et al. Gene expression profiling reveals a conserved microglia signature in larval zebrafish. Glia. 2020;68:298-315.

12. Kuil LE, Oosterhof N, Geurts SN, van der Linde HC, Meijering E, van Ham TJ. Reverse genetic screen reveals that Il34 facilitates yolk sac macrophage distribution and seeding of the brain. Dis Model Mech. 2019;12:dmm037762.

13. Wu S, Xue R, Hassan S, Nguyen TML, Wang T, Pan H, et al. Il34-Csf1r pathway regulates the migration and colonization of microglial precursors. Dev Cell. 2018;46:552-563e554.

14. Travis MA, Sheppard D. TGF-beta activation and function in immunity. Annu Rev Immunol. 2014;32:51-82.

15. Lund H, Pieber M, Parsa R, Grommisch D, Ewing E, Kular L, et al. Fatal demyelinating disease is induced by monocyte-derived macrophages in the absence of TGF-beta signaling. Nat Immunol. 2018;19:1-7.

16. Zoller T, Schneider A, Kleimeyer C, Masuda T, Potru PS, Pfeifer $\mathrm{D}$, et al. Silencing of TGFbeta signalling in microglia results in impaired homeostasis. Nat Commun. 2018;9:4011.

17. Derenzini M, Montanaro L, Trere D. Ribosome biogenesis and cancer. Acta Histochem. 2017;119:190-7.

18. Mills EW, Green R. Ribosomopathies: There's strength in numbers. Science. 2017;358:eaan2755.

19. Morcelle C, Menoyo S, Moron-Duran FD, Tauler A, Kozma SC, Thomas G, et al. Oncogenic MYC induces the impaired ribosome biogenesis checkpoint and stabilizes p53 independent of increased ribosome content. Cancer Res. 2019;79:4348-59.

20. Berghmans S, Murphey RD, Wienholds E, Neuberg D, Kutok JL, Fletcher $\mathrm{CD}$, et al. tp53 mutant zebrafish develop malignant peripheral nerve sheath tumors. Proc Natl Acad Sci USA. 2005;102:407-12.

21. Xu J, Wang T, Wu Y, Jin W, Wen Z. Microglia colonization of developing zebrafish midbrain is promoted by apoptotic neuron and lysophosphatidylcholine. Dev Cell. 2016;38:214-22. 
22. Oosterhof N, Kuil LE, van der Linde HC, Burm SM, Berdowski W, van Ijcken WFJ, et al. Colony-stimulating factor 1 receptor (CSF1R) regulates microglia density and distribution, but not microglia differentiation in vivo. Cell Rep. 2018;24:1203-1217e1206.

23. Nguyen-Chi M, Laplace-Builhe B, Travnickova J, Luz-Crawford P, Tejedor G, Phan QT, et al. Identification of polarized macrophage subsets in zebrafish. Elife. 2015;4:e07288.

24. Lu XJ, Chen Q, Rong YJ, Chen F, Chen J. CXCR3.1 and CXCR3.2 differentially contribute to macrophage polarization in teleost fish. J Immunol. 2017;198:4692-706.

25. Butovsky O, Jedrychowski MP, Moore CS, Cialic R, Lanser AJ, Gabriely $\mathrm{G}$, et al. Identification of a unique TGF-beta-dependent molecular and functional signature in microglia. Nat Neurosci. 2014;17:131-43.

26. Buttgereit A, Lelios I, Yu X, Vrohlings M, Krakoski NR, Gautier EL, et al. Sall1 is a transcriptional regulator defining microglia identity and function. Nat Immunol. 2016;17:1397-406.

27. Sweet T, Khalili K, Sawaya BE, Amini S. Identification of a novel protein from glial cells based on its ability to interact with NFkappaB subunits. J Cell Biochem. 2003;90:884-91.

28. Sweet T, Sawaya BE, Khalili K, Amini S. Interplay between NFBP and NF-kappaB modulates tat activation of the LTR. J Cell Physiol. 2005;204:375-80.

29. Dyson HJ, Komives EA. Role of disorder in IkappaB-NFkappaB interaction. IUBMB Life. 2012;64:499-505.

30. Stark LA, Dunlop MG. Nucleolar sequestration of RelA (p65) regulates NF-kappaB-driven transcription and apoptosis. Mol Cell Biol. 2005;25:5985-6004.

31. Daroczi B, Kari G, Ren Q, Dicker AP, Rodeck U. Nuclear factor kappaB inhibitors alleviate and the proteasome inhibitor PS-341 exacerbates radiation toxicity in zebrafish embryos. Mol Cancer Ther. 2009;8:2625-34.

32. Wang T, Yan B, Lou L, Lin X, Yu T, Wu S, et al. Nlrc3-like is required for microglia maintenance in zebrafish. J Genet Genomics. 2019;46:291-9.

33. Don EK, Formella I, Badrock AP, Hall TE, Morsch M, Hortle E, et al. A Tol 2 gateway-compatible toolbox for the study of the nervous system and neurodegenerative disease. Zebrafish. 2017;14:69-72.

34. Harvie EA, Green JM, Neely MN, Huttenlocher A. Innate immune response to Streptococcus iniae infection in zebrafish larvae. Infect Immun. 2013;81:110-21.

35. Kanther M, Sun X, Muhlbauer M, Mackey LC, Flynn EJ 3rd, Bagnat M, et al. Microbial colonization induces dynamic temporal and spatial patterns of NF-kappaB activation in the zebrafish digestive tract. Gastroenterology. 2011;141:197-207.

36. Baeuerle PA, Henkel T. Function and activation of NF-kappa B in the immune system. Annu Rev Immunol. 1994;12:141-79.

37. Barnes PJ, Karin M. Nuclear factor-kappaB: a pivotal transcription factor in chronic inflammatory diseases. N. Engl J Med. 1997;336:1066-71.

38. Grinberg-Bleyer Y, Oh H, Desrichard A, Bhatt DM, Caron R, Chan TA, et al. NF-kappaB c-Rel is crucial for the regulatory $\mathrm{T}$ cell immune checkpoint in cancer. Cell. 2017;170:1096-1108e1013.

39. De Siervi A, De Luca P, Moiola C, Gueron G, Tongbai R, Chandramouli GV, et al. Identification of new Rel/NFkappaB regulatory networks by focused genome location analysis. Cell Cycle. 2009;8:2093-100.

40. Hase Y, Horsburgh K, Ihara M, Kalaria RN. White matter degeneration in vascular and other ageing-related dementias. J Neurochem. 2018;144:617-33.

41. Skripuletz T, Hackstette D, Bauer K, Gudi V, Pul R, Voss E, et al. Astrocytes regulate myelin clearance through recruitment of microglia during cuprizone-induced demyelination. Brain. 2013;136:147-67.
42. Lampron A, Larochelle A, Laflamme N, Prefontaine P, Plante MM, Sanchez MG, et al. Inefficient clearance of myelin debris by microglia impairs remyelinating processes. J Exp Med. 2015;212:481-95.

43. Ginhoux F, Greter M, Leboeuf M, Nandi S, See P, Gokhan S, et al. Fate mapping analysis reveals that adult microglia derive from primitive macrophages. Science. 2010;330:841-5.

44. Ferrero G, Mahony CB, Dupuis E, Yvernogeau L, Di Ruggiero E, Miserocchi M, et al. Embryonic Microglia derive from primitive macrophages and are replaced by cmyb-dependent definitive microglia in zebrafish. Cell Rep. 2018;24:130-41.

45. Robertson IB, Rifkin DB. Regulation of the bioavailability of TGF-beta and TGF-beta-related proteins. Cold Spring Harb Perspect Biol. 2016;8:a021907.

46. Shull MM, Ormsby I, Kier AB, Pawlowski S, Diebold RJ, Yin M, et al. Targeted disruption of the mouse transforming growth factor-beta 1 gene results in multifocal inflammatory disease. Nature. 1992;359:693-9.

47. Chen X, Kandasamy K, Srivastava RK. Differential roles of RelA (p65) and c-Rel subunits of nuclear factor kappa B in tumor necrosis factor-related apoptosis-inducing ligand signaling. Cancer Res. 2003;63:1059-66.

48. Pizzi M, Goffi F, Boroni F, Benarese M, Perkins SE, Liou HC, et al. Opposing roles for NF-kappa B/Rel factors p65 and c-Rel in the modulation of neuron survival elicited by glutamate and interleukin-1beta. J Biol Chem. 2002;277:20717-23.

49. Pohl T, Gugasyan R, Grumont RJ, Strasser A, Metcalf D, Tarlinton D, et al. The combined absence of NF-kappa B1 and c-Rel reveals that overlapping roles for these transcription factors in the $\mathrm{B}$ cell lineage are restricted to the activation and function of mature cells. Proc Natl Acad Sci USA. 2002;99:4514-9.

50. Tanabe S, Yamashita T. B-1a lymphocytes promote oligodendrogenesis during brain development. Nat Neurosci. 2018;21:506-16.

51. Ferrero G, Gomez E, Lyer S, Rovira M, Miserocchi M, Langenau $\mathrm{DM}$, et al. The macrophage-expressed gene (mpeg) 1 identifies a subpopulation of B cells in the adult zebrafish. J Leukoc Biol. 2020;107:431-43.

52. Kimmel CB, Ballard WW, Kimmel SR, Ullmann B, Schilling TF. Stages of embryonic development of the zebrafish. Dev Dyn. 1995;203:253-310.

53. Jin $\mathrm{H}$, Li L, Xu J, Zhen F, Zhu L, Liu PP, et al. Runx1 regulates embryonic myeloid fate choice in zebrafish through a negative feedback loop inhibiting Pu.1 expression. Blood. 2012;119:5239-49.

54. Ellett F, Pase L, Hayman JW, Andrianopoulos A, Lieschke GJ. mpeg1 promoter transgenes direct macrophage-lineage expression in zebrafish. Blood. 2011;117:e49-56.

55. Renshaw SA, Loynes CA, Trushell DM, Elworthy S, Ingham PW, Whyte MK. A transgenic zebrafish model of neutrophilic inflammation. Blood. 2006;108:3976-8.

56. Page DM, Wittamer V, Bertrand JY, Lewis KL, Pratt DN, Delgado $\mathrm{N}$, et al. An evolutionarily conserved program of B-cell development and activation in zebrafish. Blood. 2013;122: e1-11.

57. Xiao A, Wang Z, Hu Y, Wu Y, Luo Z, Yang Z, et al. Chromosomal deletions and inversions mediated by TALENs and CRISPR/Cas in zebrafish. Nucleic Acids Res. 2013;41:e141.

58. Kawakami K, Shima A, Kawakami N. Identification of a functional transposase of the Tol2 element, an Ac-like element from the Japanese medaka fish, and its transposition in the zebrafish germ lineage. Proc Natl Acad Sci USA. 2000;97:11403-8.

59. Hinits Y, Osborn DP, Hughes SM. Differential requirements for myogenic regulatory factors distinguish medial and lateral somitic, cranial and fin muscle fibre populations. Development. 2009;136:403-14. 
60. Crowhurst MO, Layton JE, Lieschke GJ. Developmental biology of zebrafish myeloid cells. Int J Dev Biol. 2002; 46:483-92.

61. Willett CE, Cherry JJ, Steiner LA. Characterization and expression of the recombination activating genes (rag1 and rag2) of zebrafish. Immunogenetics. 1997;45:394-404.
62. Finckbeiner S, Ko PJ, Carrington B, Sood R, Gross K, Dolnick B, et al. Transient knockdown and overexpression reveal a developmental role for the zebrafish enosflb gene. Cell Biosci. 2011;1:32.

63. Link V, Shevchenko A, Heisenberg CP. Proteomics of early zebrafish embryos. BMC Dev Biol. 2006;6:1. 